\title{
Validation of HPPCALC
}

\author{
Jeffrey R. Belt
}

October 2006

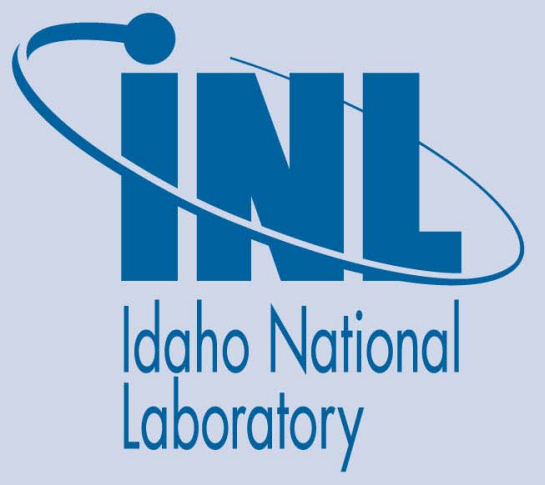

The INL is a U.S. Department of Energy National Laboratory operated by Battelle Energy Alliance 
INL/EXT-06-11848

\section{Validation of HPPCALC}

Jeffrey R. Belt

October 2006

\section{Idaho National Laboratory \\ Idaho Falls, Idaho 83415}

Prepared for the

U.S. Department of Energy

Office of Nuclear Energy

Under DOE Idaho Operations Office

Contract DE-AC07-05ID14517 


\begin{abstract}
HPPCALC 2.1 was developed to analyze the raw data from a PNGV Hybrid Pulse Power Characterization (HPPC) test and produce seven standard plots that consist of resistance, power and available energy relationships. The purpose of the HPPC test is to extrapolate the total power capability within predetermined voltage limits of a prototype or full production cell regardless of chemistry with respect to the PNGV goals as outlined in the PNGV Testing Manual, Revision 3. The power capability gives the Electrochemical Energy Storage team the tools to compare different battery sizes and chemistries for possible use in a hybrid electric vehicle.

The visual basic program HPPCALC 2.1 opens the comma separated value file that is produced from a Maccor, Bitrode, or Energy Systems tester. It extracts the necessary information and performs the appropriate calculations. This information is arranged into seven graphs: Resistance versus Depth of Discharge, Power versus Depth of Discharge, Power versus Energy, Energy versus Power, Available Energy versus Power, Available Energy versus Power, and Power versus Depth of Discharge. These are the standard plots that are produced for each HPPC test.

The primary metric for the HPPC test is the PNGV power, which is the power at which the available energy is equal to $300 \mathrm{Wh}$. The PNGV power is used to monitor the power degradation of the battery over the course of cycle or calendar life testing.
\end{abstract}




\section{General Information for HPPCALC-XL Version 2.1}

\section{Program Installation \& Setup}

HPPCALC-XL runs under Windows 95, Windows 98, Windows NT, and Windows 2000. It will not run under earlier versions of Windows.

To install the program, run SETUP.EXE (from the installation floppy disk or CDROM, whichever is provided) and follow the prompts. This can be done just as other Windows programs are installed, either by using the RUN command on the Start Menu or by double-clicking on SETUP.EXE in a Windows Explorer window. The default installation directory for the program is Program Files LHPPCALC-XL. This directory can be changed by the user during installation.

The first time the program is installed on a given computer, the installation routine may need to update some Windows system files. If this happens, the SETUP program will need to be restarted by the user after these files are updated. (No undesirable side effects have been noted from updating these files on a number of computers, but it is not possible to test all possible configurations.)

\section{Uninstalling the Program}

The program can be uninstalled in the same fashion as other well-behaved Windows programs, by opening the Windows Control Panel and using the Add/Remove Programs option. Note that it treats all required Windows system files as shared files, so these will not be removed if they have been installed previously by some other program. If there are results files generated by the program which are still in the program directory, neither these nor the directory can be removed by Windows; the user must delete these files manually.

\section{Starting the Program}

HPPCALC-XL will be installed on your Windows Start Menu as "INEEL HPPC Calculation Program". It can also be started by double-clicking on HPPCALC-XL. EXE in an Explorer window. Note that several files other than HPPCALC-XL.EXE are installed in the program directory. These files must remain in the same directory as the executable program file in order for the program to work properly.

\section{Description of Program Operation During Use}

1. A title screen with the program name and INEEL logo will appear when the program starts. If you click on this screen, the program will continue immediately; otherwise program execution continues after about 6 seconds. See Appendix A

2. A file selection menu will be displayed. Choose an HPPC data file to be processed, by navigating through the directory structure if necessary and clicking on the selected file. (If this data file is in UNIX text file format without DOS-type line delimiters, there will be a short delay while the program creates a temporary file with line delimiters. This temporary file will be deleted after the program has finished with it.)

3. A User Input form with several sections will be displayed, See Appendix B. The name of the selected input data file is displayed for information. The allowed or required user choices are as follows:

a. Select Data File Type (MANDATORY) - select one of the available choices. For Version 2.1, these include the following types of comma-separated-value (CSV) files: 
1. "ADAPT complete data file" - these are files which have been downloaded from the links provided in the INEEL ADAPT data file archive system from the corresponding individual data file pages.

2. "Downloaded ADAPT .CSV file" - these are files which have been downloaded from the INEEL ADAPT screen which allows selecting individual variables. These files must include all the variables shown in the ADAPT variable list down to "Mode", EXCEPT for the "record number." (Note that these are commonly in UNIX text file format.)

3. "Maccor raw data file" - these are text (not binary) files which are produced as direct output from Maccor cell testers. They can be obtained either directly from the test laboratory or by downloading them from the ADAPT "tote sheet" pages for the cell or battery of interest.

4. "Bitrode .CSV file" -- these are raw data files in CSV format which are produced by the INEEL Bitrode module testers.

5. "Energy Systems" -- these are raw data files in CSV format which are produced by the INEEL Energy Systems testers.

Because these file types are generally specific to particular types of INEEL testers, Option (3) is the only one of these choices which is likely to be useable by non-INEEL users at this time. For the convenience of potential users who only wish to see how this program works, a few sample data files of types (1) and (3) have been provided on the installation disk. These are non-proprietary data files derived from DOE-sponsored testing, i.e., they are NOT Protected Battery Information.

b. Select Battery Type (MANDATORY) - select one of the available choices which describes the type of cell or battery whose HPPC data is being analyzed. Selection is done by simply clicking on a given line so that it is highlighted. The purpose for this selection is to determine the minimum and maximum voltage to be used for calculations. Additionally, the type of cell or battery is automatically included in the title for each of the seven output graphs.

If the data file is not an instance of any of the available choices, the user must exit the program and add an appropriate description and minimum and maximum voltage to the text file MAXVOLTS.DAT, which is installed in the same directory with the program. When this modified file has been saved, the new entry will appear in the list box the next time the program is run. (For Version 2.1, this text file is limited to a total of 50 battery types.)

c. Short Description or Comment (OPTIONAL) - can be typed in the text box provided. This text will then be saved with the calculation results for user information. Note that the program will automatically save the name of the file processed, the data and time processed, and a number of parameter values such as the max and min voltages used for calculations. This short description is added in parenthesis to the chart title for all seven output graphs.

d. After these selections are made, clicking on "Proceed" allows the program to continue processing the data file. Clicking on "Cancel" instead will terminate the program execution.

e. The button labeled "Display Data File Viewer" will bring up a simple text viewer showing the first few (typically 100 to 200 ) lines in the selected data file. The file can be paged through using "Next" and "Previous" buttons. This viewer must be closed before returning to the user input form, because the data file is open while the text is displayed. Note that the viewer actually displays blocks of 10,000 characters, so the first and/or last lines displayed may be incomplete. 
4. If program execution is successful, a "Calculations Completed" screen will appear. This screen initially has 4 visible buttons, as follows:

a. "WRITE to Excel Spreadsheet" will copy the calculated results to an Excel 97 or 2000 spreadsheet template and save the resulting spreadsheet with the same name as the original data file and an "XLS" extension. The file will be saved in the directory where HPPCALC-XL is installed. (This choice was made so that the program can be run using data files on CD-ROM disks or other read-only devices.)

b. "WRITE to Text File" will save the calculated results to a text file with the same name as the original data file and a "TXT" extension. The file will be saved in the directory where HPPCALC-XL is installed. The file will be in CSV format (with quoted strings) so that it can be manually loaded into a spreadsheet if desired.

c. "QUIT" will exit the program. If the results have not yet been saved either as a spreadsheet or a text file, the user will be asked to confirm this choice.

d. "Process ANOTHER FILE" will return to the data file selection screen, where the user can choose another file to be processed. If the results have not yet been saved, the user will be asked to confirm this choice.

e. If the data is saved to a spreadsheet, another button labelled "VIEW Excel Spreadsheet" will appear. Clicking this button will open a copy of Excel and display the spreadsheet which was just saved. This copy of Excel is not linked to the program, so it must/can be closed independently of HPPCALC-XL. Note that the spreadsheet will appear to contain only graphs of the calculated results; the numerical values calculated by the program are on a worksheet named "data" which is initially hidden. The unscaled the numerical values calculated by the program are on a worksheet named "unscaled data" which is initially hidden. The values extracted from the data, which can be used to verify the results of the program are on a worksheet named "lookup" which is initially hidden. This worksheet can be displayed if desired by using the Excel "Format/Sheet/Unhide" menu.

\section{Data File Constraints \& Requirements}

Data files to be processed must conform to one of the defined formats described above. In addition, data files must generally contain at least one data record from the rest period just prior to the start of the HPPC discharge (i.e. $\mathrm{C} / 1$ discharge). This is primarily because the program uses this step as a flag to find the beginning of the test data (and to ignore all the unused header and data information which some data files contain.) If this initial data record prior to the start of discharge is not present, the data file can be manually edited before processing to add a dummy "Rest" record just before the first discharge step begins. Such a dummy record must contain the appropriate number of numeric values, separated by commas, up to and including units for the temperature "C". The numeric values can be all zeroes.

Additional data file formats could be added to the program if needed. However, this will require recompiling and re-distributing the program. While it might have been possible to generalize the program such that arbitrary data file formats could be either determined automatically or defined by the user, this was not considered cost-effective for Version 2.1 due to the high degree of program complexity required. The program generally assumes that the data was acquired in accordance with the PNGV Testing Manual. A few common procedural errors are detected and indicated to the user, specifically those resulting from premature termination of the HPPC test. Processing will generally continue in these cases, but the resulting output should be carefully inspected for non-sensible results, especially the final values for data files that terminate prematurely.

Note that data file formats, especially for the Maccor testers, have been subject to a number of revisions over time as the tester manufacturers modify their system software. The number and order of data file variables required for this program to operate correctly is based on software versions in use at INEEL as of August 1999.

\section{Other Program Constraints \& Requirements}


Use of the "WRITE Excel Spreadsheet" and "VIEW Excel Spreadsheet" output options requires the user to have Excel 97 installed on his/her computer. The "WRITE to Text File" option can be used if the correct version of Excel is not available. The resulting CSV file should be able to be loaded into almost any spreadsheet program. In particular, if the file is loaded into Excel 97, it will produce a data worksheet which is identical to that produced by the Excel Write option. The resulting data can be graphed or otherwise manipulated as desired by the user.

If the "WRITE Excel Spreadsheet" option is used and a previous version of the output spreadsheet exists in the target directory, Excel will ask whether it should be overwritten. If the Excel output option is chosen when a copy of the output spreadsheet is already open in Excel, the program will fail. If Excel is already open with a different spreadsheet, the "VIEW" option will open in another window of this existing copy of Excel.

The program does not check for available disk space, and it overwrites any previous versions of output text files without warning. It will not modify or overwrite any input data files (unless these are deliberately given .TXT extensions and stored in the same directory as the program executable, which is highly unlikely.)

As noted previously, output files are written to the directory where the program is installed (normally C: (Program Files 1 HPPCALC-XL, unless this is changed by the user during installation.) This prevents running the program directly from a CD-ROM drive, although the data files to be processed can be on such a drive. (This limitation could possibly be removed in future versions at the expense of some program complexity.)

\section{Information Regarding Program Calculations}

In general the calculations made by this program are intended to be a direct implementation of the corresponding equations and methods defined in Revision 2 of the PNGV Testing Manual. Any observed deviations should be reported to the author, Jeffrey R. Belt at beltjr@inel.gov, or to the INEEL program manager Chester Motloch at motlcg@inel.gov.

The program makes a number of assumptions about the data files in order to avoid having the user supply information manually. In particular, it assumes that the PNGV HPPC test procedure has been strictly followed, including the use of $10 \%$ DOD discharge intervals (including the pulse profiles). However, it will adjust if a different discharge interval is actually used. The capacity removed during the initial $\mathrm{C} / 1$ discharge period is assumed to be $10 \%$ DOD, and all succeeding DOD calculations are based on this value. This assumption is made because the total capacity removed is not normally accumulated in INEEL HPPC data files, i.e., the A-h variable is typically reset at the end of each $10 \%$ DOD interval.

The use of $18 \mathrm{~s}$ discharge pulses and $2 \mathrm{~s}$ initial regen pulses is required, and data must be available at about these times in each profile. If a data point is available at about $2 \mathrm{~s}$ into the discharge pulse, the program also calculates the $2 \mathrm{~s}$ discharge resistances and power capability; however, this is not mandatory. All pulse data points are ignored except those used for the calculations, i.e., those immediately prior to the start of discharge and regen pulses and the $2 \mathrm{~s}$ and $18 \mathrm{~s}$ (discharge only) points during the discharge and first regen pulse in each test profile. The resistances are calculated at 12 seconds during discharge and 10 seconds for the regen for dual mode applications.

The program verifies only the length of the pulses; it does not verify the shape of the pulse profile, nor does it verify that current is constant during the pulses. This allows it to be used with HPPC data files based on either the current-step or power-step versions of the HPPC test profile (Power Assist or Dual Mode versions). However, this means that it may calculate resistances and power capabilities for pulses where voltage limiting occurs. Strictly speaking, these calculated results are not 'correct'. The data points where this condition has been observed to date generally do not deviate excessively from the normal resistance and power curves, but the user should be aware of this behavior. 
Pulse power capabilities are calculated according to the PNGV Testing Manual formulas, using local linearly interpolated OCV values for regen power capability. ("Local linear interpolation" refers to the use of the $\mathrm{OCV}$ values for the previous and next discharge pulses in the test sequence.) If regen data points exist at higher DOD values than the last discharge OCV point, the last 2 discharge OCV points will be extrapolated. In both this case and the available energy calculations discussed below, such extrapolation can result in the calculated values differing significantly from the "best fit". However, these generally occur at the extremes of DOD where the cell behavior is both less critical and not so well characterized due to other limitations of the HPPC test.

Available energy is calculated as the difference between the discharge and regen energy vs power curves, with values linearly interpolated between the points of both curves (i.e., no polynomial or other curvefitting is used.) See Appendix E of Revision 3 of the PNGV Testing Manual for more information on this calculation. Available Energy calculations are done for both Power Assist and Dual Mode regen-todischarge power targets. The available energy calculations are only performed over a power range whose upper limit is the Pulse Power Limit (where Available Energy goes to zero) and whose lower limit is the last useable data point on the discharge or regen curves (whichever is higher). This means that the program does not extrapolate Available Energy outside the HPPC data points (although it will extrapolate the curves to find the corresponding Pulse Power Limit.) This limitation can occasionally result in Available Energy being calculated over a very small range of power values, or in extreme cases not at all.

The Pulse Power Limit (a single-value metric of pulse power capability) is calculated based on linear interpolation between points on both the discharge and regen power capability curves. Note that the Pulse Power Limit reported by the program is calculated using the actual regen power capability curve, i.e., it is effectively based on a regen-to-discharge power ratio of 1.0 rather than the 30/25 and 40/65 ratios used for Available Energy calculations. (However, the appropriate pulse power limit values based on scaled regen power requirements are used for calculating Available Energy.) Note that Pulse Power Limit does not relate directly to the PNGV program power goals. It is a reporting convenience which is used primarily for laboratory cells, which may be difficult or premature to scale to full-size batteries for comparison with the PNGV power and energy goals.

For cells that use a battery scaling factor, the PNGV pulse power is calculated. This value is the scaled power at which the available energy equals the PNGV energy goal, $300 \mathrm{Wh}$. 


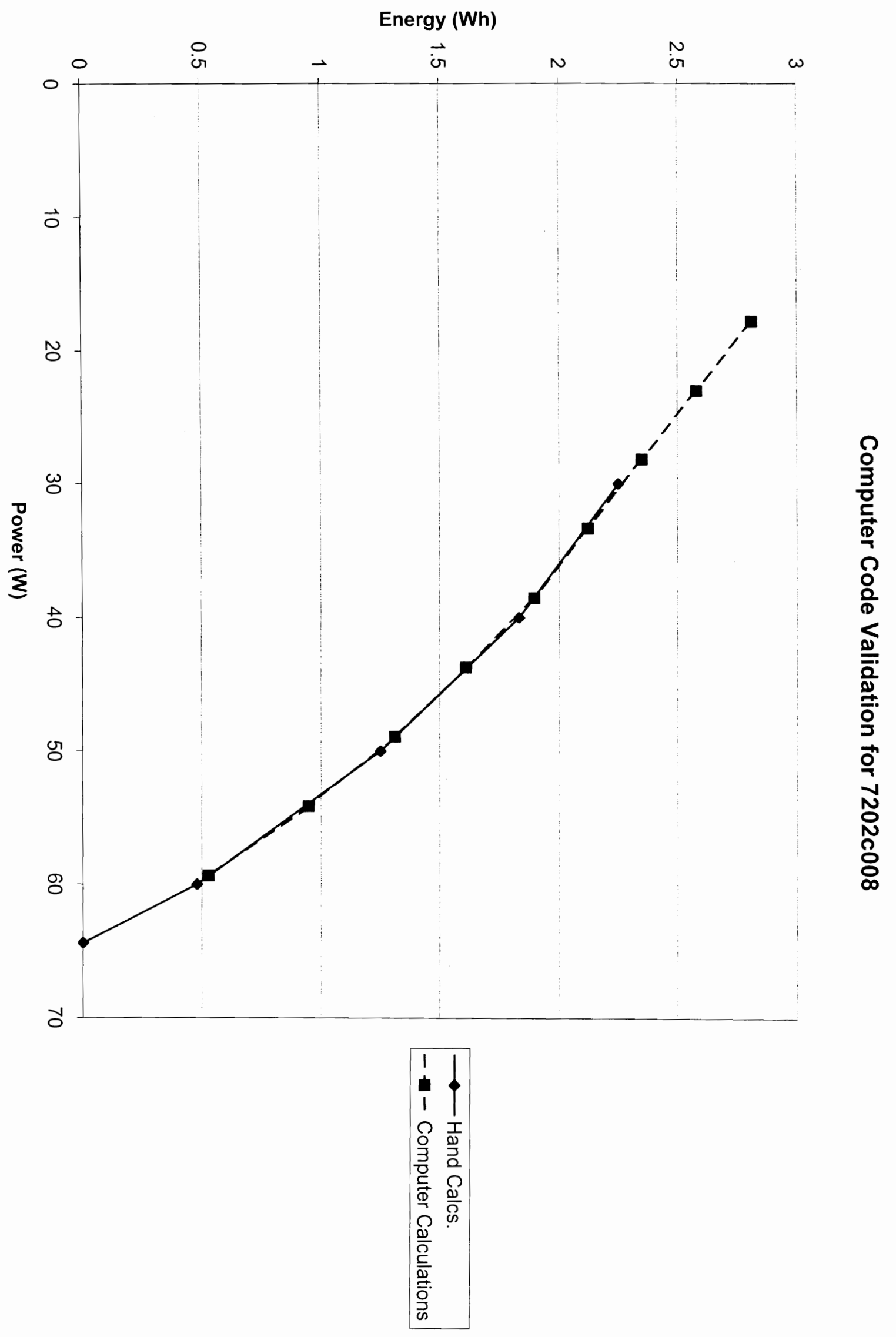




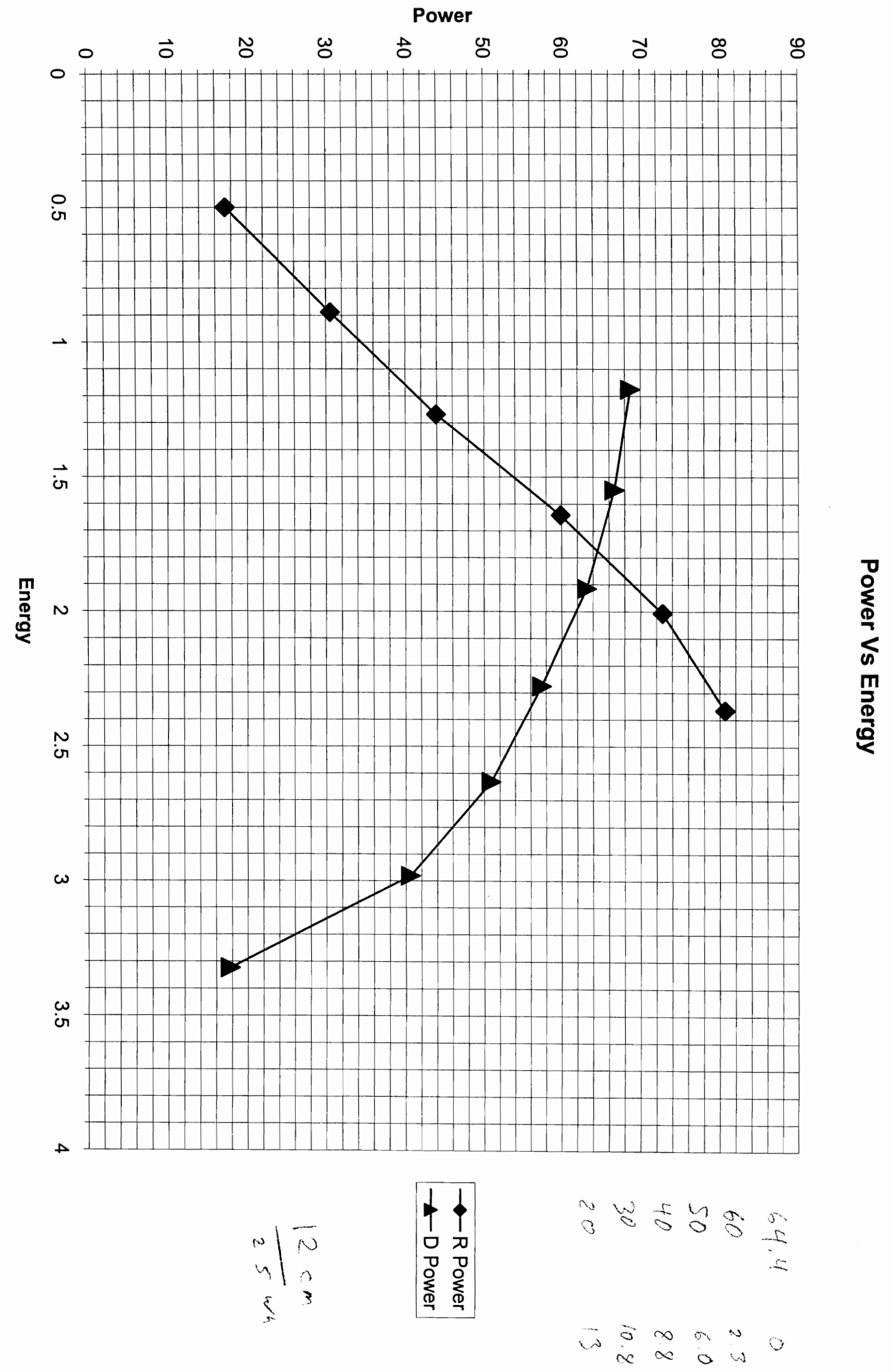




\begin{tabular}{|c|c|c|c|c|c|c|c|c|}
\hline \multicolumn{6}{|c|}{$F$} & \multirow{2}{*}{\multicolumn{2}{|c|}{$59.11596 \quad 53.24059$}} & \\
\hline \multicolumn{6}{|c|}{ Input File: $7202 \mathrm{c} 008.002$} & & & \\
\hline \multicolumn{6}{|c|}{ Comment: P72 ATD Gen. 21 Ah Lilon Cell 02 () } & & \multicolumn{2}{|l|}{41.40935} \\
\hline \multirow{2}{*}{\multicolumn{3}{|c|}{ Processing Date/Time: }} & \multirow{2}{*}{\multicolumn{2}{|c|}{ 2/23/2001 \#\#\#\#\# }} & & \multirow{2}{*}{\multicolumn{3}{|c|}{$\begin{array}{rr}0.545686 & 1.365143 \\
549767 & 1098786\end{array}$}} \\
\hline & & & & & & & \multicolumn{2}{|l|}{1098.786} \\
\hline \multirow{3}{*}{$\begin{array}{r}10 \% \text { DOD } \\
0.100001\end{array}$} & Max V & Min V & Idis Final & PPL & \multicolumn{4}{|c|}{ \% DODppl Scaling FarPA PPL } \\
\hline & \multirow{2}{*}{\multicolumn{2}{|c|}{4.1}} & 4.999619 & 66.71679 & 39.81802 & 0 & \multirow{2}{*}{\multicolumn{2}{|c|}{$\begin{array}{r}64.54161 \\
936.026\end{array}$}} \\
\hline & & & & & & 435.9155 & & \\
\hline \multirow{8}{*}{$\begin{array}{l}1 \\
2 \\
3 \\
4 \\
5 \\
6\end{array}$} & OCV & \multirow[t]{2}{*}{ Rdis } & \multirow[t]{2}{*}{ Pdis } & \multirow[t]{2}{*}{ WHdis } & \multirow[t]{2}{*}{ Rdis2s } & \multirow[t]{2}{*}{ Pdis2s } & \multirow[t]{2}{*}{ Rdis12s } & \multirow[t]{2}{*}{ Pdis12s } \\
\hline & 4.099641 & & & & & & & \\
\hline & 4.008545 & 0.038209 & 79.18731 & 0.400725 & 0.023194 & 130.4508 & 0.033336 & 90.7624 \\
\hline & 3.91928 & 0.036925 & 74.68672 & 0.791936 & 0.022827 & 120.8169 & 0.032591 & 84.6198 \\
\hline & 3.848936 & 0.037081 & 68.68255 & 1.174542 & 0.022768 & 111.8581 & 0.0328 & 77.64699 \\
\hline & 3.761044 & 0.03424 & 66.68035 & 1.549182 & 0.021606 & 105.6714 & 0.030387 & 75.13531 \\
\hline & 3.682002 & 0.03238 & 63.18798 & 1.916051 & 0.02051 & 99.75412 & 0.028442 & 71.93486 \\
\hline & 3.627985 & 0.032809 & 57.42124 & 2.276631 & 0.020509 & 91.86044 & 0.028773 & 65.47677 \\
\hline 69.99999 & 3.583429 & 0.034395 & 50.88758 & 2.631761 & 0.02112 & 82.87314 & 0.029932 & 58.47454 \\
\hline 79.99999 & 3.52697 & 0.038973 & 40.56421 & 2.98159 & 0.022981 & 68.79211 & 0.033611 & 47.03597 \\
\hline 89.99999 & 3.435264 & 0.073493 & 17.76754 & 3.32334 & 0.031711 & 41.1783 & 0.056424 & 23.14237 \\
\hline DOD & OCV (inter) & Rregen & Pregen & WHregen & Rregen Du & Pregen Du & Vreginit & \\
\hline 12.49807 & 3.986246 & 0.0225 & 20.72824 & 0.499338 & 0.031533 & 14.79062 & 3.967041 & \\
\hline 22.49891 & 3.901701 & 0.022177 & 36.66058 & 0.888292 & 1495 & 25.814 & 3.8 & \\
\hline 32.49955 & 3.826967 & 0.02124 & 52.70411 & 1.268955 & 0.032308 & 34.64927 & 9802 & \\
\hline 42.49992 & 3.741284 & 0.020469 & 71.8518 & 1.641562 & 0.029055 & 50.6183 & 3.7 & \\
\hline 52.50003 & 3.668498 & 0.020266 & 87.29893 & 2.006757 & 0.028241 & 62.64404 & 3.6 & \\
\hline 62.50003 & 3.616846 & 0.020468 & 96.78207 & 2.365885 & 0.028403 & 69.74409 & 3.598993 & \\
\hline 72.50002 & 3.569314 & 0.021122 & 103.0105 & 2.719772 & 0.029587 & 73.53843 & 3.55108 & \\
\hline 82.50002 & 3.504043 & 0.023276 & 104.9773 & 3.067966 & 0.032147 & 76.00893 & 3.486534 & \\
\hline 92.50002 & 3.412337 & 0.029134 & 96.7748 & 3.406867 & 0.038818 & 72.63186 & 3.387198 & \\
\hline Pwr 30 & $\mathrm{AE} 30 / 25$ & Pwr 35 & AE 35/45 & & Pdis $30 / 25$ & Pdis $35 / 45$ & & \\
\hline 17.76754 & 2.80953 & 23.14237 & 2.710774 & & 17.27354 & 19.01651 & & \\
\hline 22.96465 & 2.579367 & 28.65412 & 2.480678 & & 30.55049 & 33.18943 & & \\
\hline 28.16178 & 2.349204 & 34.16587 & 2.244658 & & 43.92009 & 44.54906 & & \\
\hline 33.35889 & 2.121352 & 39.67762 & 1.981124 & & 59.8765 & 65.08067 & & \\
\hline 38.55602 & 1.895467 & 45.18937 & 1.727426 & & 72.74911 & 80.54233 & & \\
\hline 43.75313 & 1.609325 & 50.70111 & 1.488895 & & 80.65172 & 89.67097 & & \\
\hline 48.95025 & 1.310994 & 56.21286 & 1.220301 & & 85.84206 & 94.54941 & & \\
\hline 54.14737 & 0.9468 & 61.72461 & 0.886272 & & 87.48109 & 97.72577 & & \\
\hline 59.34449 & 0.527236 & 67.23636 & 0.485908 & & 80.64567 & 93.38382 & & \\
\hline 64.54161 & & 72.74811 & 0.000166 & & & & & \\
\hline
\end{tabular}




\begin{tabular}{|c|c|c|c|c|c|c|c|c|}
\hline HPPC Rest & ults file crea & ated by: & Project1 & & & Assist & Dual & \\
\hline Input File: & $7202 c 008.0$ & 002 & & & Pdis Goal & 59.116 & 53.241 & \\
\hline Comment: & P72 ATD G & Sen. $21 \mathrm{Ah}$ & Lilon Cell 0 & 20 & Preg Goal & 70.939 & 41.409 & \\
\hline Processing & Date/Time: & & 02/23/01 & 8:03 PM & E Goal & 0.546 & 1.365 & \\
\hline & & & & & \# Cells & 549.767 & 1098.786 & \\
\hline $10 \%$ DOD & Max V & Min V & Idis Final & PPL & $\%$ DODppl & Scaling Fa & PA PPL & \\
\hline 0.100001 & 4.10 & 3.00 & 4.999619 & 66.71679 & 39.81802 & $\begin{array}{r}550.0 \\
435.916\end{array}$ & $\begin{array}{r}64.54161 \\
936.026\end{array}$ & \\
\hline DOD & OCV & Rdis & Pdis & WHdis & Rdis2s & Pdis2s & Rdis12s & Pdis 12s \\
\hline 0 & 4.0996 & & & & & & & \\
\hline 10 & 4.008545 & 0.038209 & 43553.02 & 220.3989 & 0.023194 & 130.4508 & 0.033336 & 49919.32 \\
\hline 20 & 3.91928 & 0.036925 & 41077.7 & 435.5647 & 0.022827 & 120.8169 & 0.032591 & 46540.89 \\
\hline 30 & 3.848936 & 0.037081 & 37775.4 & 645.998 & 0.022768 & 111.8581 & 0.0328 & 42705.84 \\
\hline 40 & 3.761044 & 0.03424 & 36674.19 & 852.05 & 0.021606 & 105.6714 & 0.030387 & 41324.42 \\
\hline 50 & 3.682002 & 0.03238 & 34753.39 & 1053.828 & 0.02051 & 99.75412 & 0.028442 & 39564.17 \\
\hline 60 & 3.627985 & 0.032809 & 31581.68 & 1252.147 & 0.020509 & 91.86044 & 0.028773 & 36012.22 \\
\hline 69.99999 & 3.583429 & 0.034395 & 27988.17 & 1447.469 & 0.02112 & 82.87314 & 0.029932 & 32161 \\
\hline 79.99999 & 3.52697 & 0.038973 & 22310.32 & 1639.874 & 0.022981 & 68.79211 & 0.033611 & 25869.78 \\
\hline 89.99999 & 3.435264 & 0.073493 & 9772.145 & 1827.837 & 0.031711 & 41.1783 & 0.056424 & 12728.3 \\
\hline DOD & OCV (inter & Rregen & Pregen & WHregen & Rregen Du & Pregen Du & Vreginit & \\
\hline 12.49807 & 3.986246 & 0.0225 & 11400.53 & 274.6361 & 0.031533 & 8134.839 & 3.967041 & \\
\hline 22.49891 & 3.901701 & 0.022177 & 20163.32 & 488.5606 & 0.031495 & 14197.7 & 3.878843 & \\
\hline 32.49955 & 3.826967 & 0.02124 & 28987.26 & 697.9255 & 0.032308 & 19057.1 & 3.799802 & \\
\hline 42.49992 & 3.741284 & 0.020469 & 39518.49 & 902.8591 & 0.029055 & 27840.06 & 3.720455 & \\
\hline 52.50003 & 3.668498 & 0.020266 & 48014.41 & 1103.716 & 0.028241 & 34454.22 & 3.651789 & \\
\hline 62.50003 & 3.616846 & 0.020468 & 353230.14 & 1301.237 & 0.028403 & 38359.25 & 3.598993 & \\
\hline 72.50002 & 3.569314 & 0.021122 & 56655.76 & 1495.874 & 0.029587 & 40446.14 & 3.55108 & \\
\hline 82.50002 & . 3.504043 & 0.023276 & 57737.52 & 1687.381 & 0.032147 & 41804.91 & 3.486534 & \\
\hline 92.50002 & 3.412337 & 0.029134 & 453226.14 & 1873.777 & 0.038818 & 39947.52 & 3.387198 & \\
\hline Pwr 30 & $A E 30 / 25$ & Pwr 35 & $A E 35 / 45$ & & Pdis $30 / 25$ & Pdis $35 / 45$ & & \\
\hline 9772.145 & 1545.241 & 12728.3 & 31490.926 & & 9500.445 & 10459.08 & & \\
\hline 12630.56 & 1418.652 & 15759.77 & $7 \quad 1364.373$ & & 16802.77 & 18254.19 & & \\
\hline 15488.98 & 1292.062 & 18791.23 & 31234.562 & & 24156.05 & 24501.99 & & \\
\hline 18347.39 & 1166.744 & 21822.69 & $\begin{array}{ll}9 & 1089.618\end{array}$ & & 32932.07 & 35794.37 & & \\
\hline 21205.81 & 1042.507 & 24854.15 & 5950.0844 & & 40012.01 & 44298.28 & & \\
\hline 24064.22 & 885.1287 & 27885.61 & 1818.8923 & & 44358.45 & 49319.03 & & \\
\hline 26922.64 & $\quad 721.0464$ & 30917.07 & $7 \quad 671.1655$ & & 47213.14 & 52002.18 & & \\
\hline 29781.05 & 520.7402 & 33948.54 & $4 \quad 487.4494$ & & 48114.6 & 53749.17 & & \\
\hline 32639.47 & 289.9799 & 36980 & 267.2495 & & 44355.12 & 51361.1 & & \\
\hline 35497.89 & & 40011.46 & 0.091136 & & & & & \\
\hline
\end{tabular}




\begin{tabular}{|c|c|c|c|c|c|c|c|c|c|}
\hline DisVolt 0 & DisAmp0 & $\begin{array}{l}\text { DisVolt1 } \\
3817502\end{array}$ & DisAmp1 & $\begin{array}{l}\text { RegVolt0 } \\
3967041\end{array}$ & RegAmp0 & RegVolt1 & RegAmp 1 & StepWh & DisWh \\
\hline $\begin{array}{r}4.000540 \\
3.91928\end{array}$ & & 3.734646 & 5.000191 & 3.878843 & & 3.962005 & 3.749905 & 0.791936 & 0.09419 \\
\hline 3.848936 & & 3.663538 & 4.999809 & 3.799802 & & 3.879454 & 3.750095 & 1.174542 & 0.092442 \\
\hline 3.761044 & & 3.589838 & 5.000191 & 3.720455 & & 3.797208 & 3.749714 & 1.549182 & 0.090527 \\
\hline 3.682002 & & 3.520104 & 5 & 3.651789 & & 3.727779 & 3.749714 & 1.916051 & 0.088751 \\
\hline 3.627985 & & 3.463951 & 4.999619 & 3.598993 & & 3.675746 & 3.749905 & 2.276631 & 0.087378 \\
\hline 3.583429 & & 3.411459 & 4.999809 & 3.55108 & & 3.630274 & 3.749332 & 2.631761 & 0.086134 \\
\hline 3.52697 & & 3.332113 & 4.999809 & 3.486534 & & 3.573816 & 3.749905 & 2.98159 & 0.08433 \\
\hline 3.435264 & & 3.067826 & 4.999619 & 3.387198 & & 3.496452 & 3.750095 & 3.32334 & 0.079652 \\
\hline
\end{tabular}

$\begin{array}{crrrrrrr}\text { PGoal302E EGoal302E PGoal404C EGoal4040 } & & & & \\ 4437.236 & 40.9591 & 5001.433 & 128.2419 & 25000 & 0 & 0 & 300 \\ 8874.472 & 81.9182 & 10002.87 & 256.4837 & 25000 & 1545.241 & 35497.89 & 300\end{array}$

$\begin{array}{llll}13311.71 & 122.8773 & 15004.3 & 384.7256\end{array}$

$\begin{array}{llll}17748.94 & 163.8364 & 20005.73 & 512.9674\end{array}$

$22186.18 \quad 204.7955 \quad 25007.16 \quad 641.2093$

$26623.42 \quad 245.7546 \quad 30008.59 \quad 769.4512$

$\begin{array}{llll}31060.65 & 286.7137 & 35010.03 & 897.693\end{array}$

$35497.89327 .6729 \quad 40011.46 \quad 1025.935$

DisVolts1 DisAmps1 DisV12sec DisA12sec RegV1Dua RegA1Dua regV2Dual regA2Dual stepAh disAH

$\begin{array}{rrrrrrrr}4.008545 & 3.838102 & 5.112917 & 3.967041 & 4.085298 & 3.749332 & 0.100001 & 0.024981 \\ 3.91928 & 3.752651 & 5.112726 & 3.878843 & 3.996948 & 3.749714 & 0.200002 & 0.024989 \\ 3.848936 & 3.681239 & 5.112726 & 3.799802 & 3.920958 & 3.750095 & 0.300004 & 0.024996 \\ 3.761044 & 3.605707 & 5.111963 & 3.720455 & 3.829404 & 3.749714 & 0.400005 & 0.024999 \\ 3.682002 & 3.536583 & 5.112726 & 3.651789 & 3.757687 & 3.749714 & 0.500006 & 0.025001 \\ 3.627985 & 3.480888 & 5.112345 & 3.598993 & 3.705501 & 3.750286 & 0.600007 & 0.025001 \\ 3.583429 & 3.430381 & 5.113108 & 3.55108 & 3.662013 & 3.749523 & 0.700008 & 0.025001 \\ 3.52697 & 3.355154 & 5.111963 & 3.486534 & 3.60708 & 3.749523 & 0.800009 & 0.025001 \\ 3.435264 & 3.146868 & 5.1112 & 3.387198 & 3.532769 & 3.749714 & 0.900011 & 0.025001\end{array}$

$$
\begin{aligned}
& .497026 \\
& .886126 \\
& 1.166984 \\
& 1.639709 \\
& 2.004803 \\
& 2.364009 \\
& 2.717895 \\
& 3.065919 \\
& 3.402992
\end{aligned}
$$




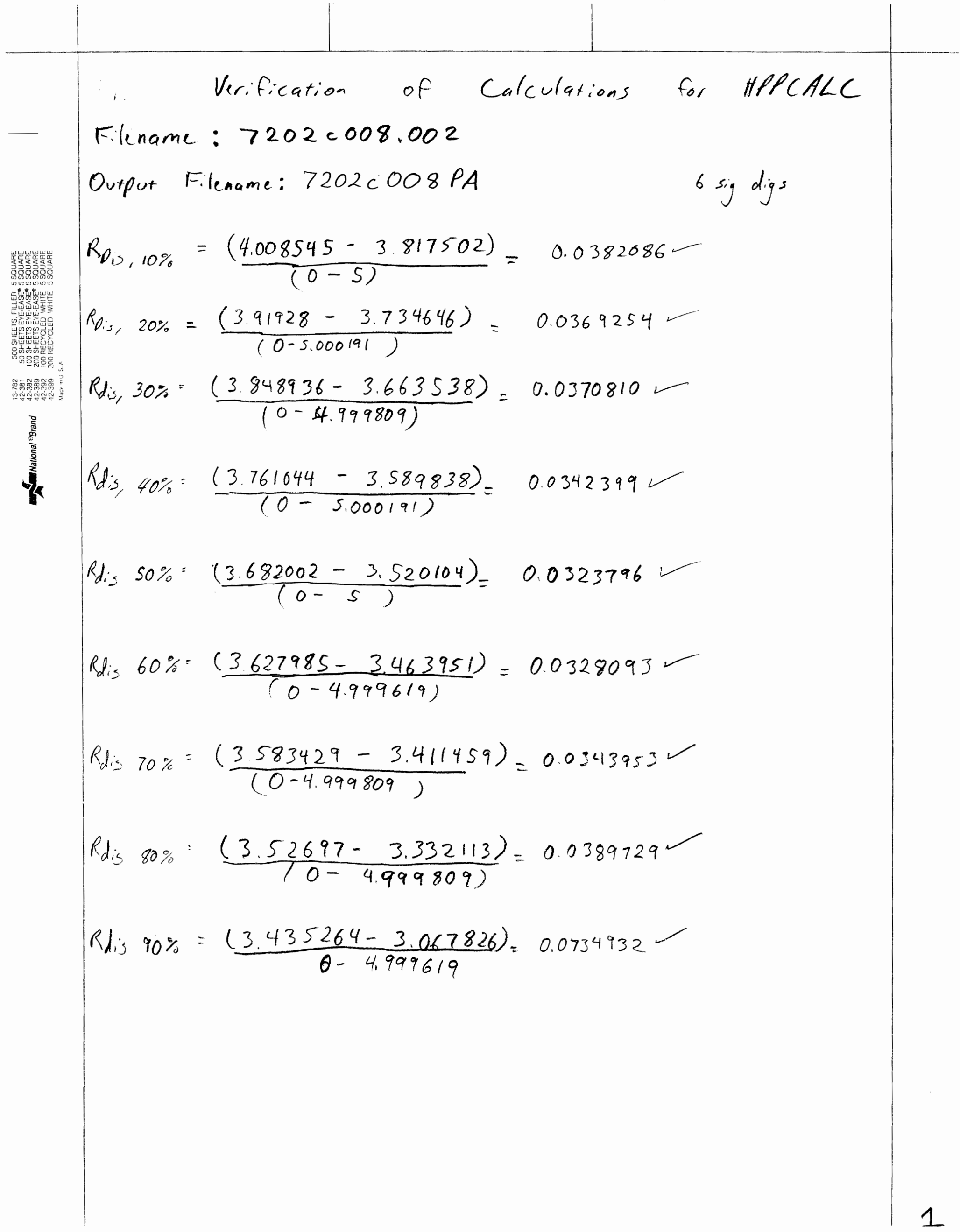


$R_{\text {reg, 10\% }}=(3.967040-4.051423) /(0-3.750286)=0.02250042$ $\operatorname{Rreg}_{120 \%}=(3.878843-3.962005) /(0-3.749905)=0.02217710$ $R_{\text {reg, } 30 \%}=(3.799802-3.879454) /(0-3.750095)=0.02124000$ Rreg, $40 \%=(3.720455-3.797208) /(0-3.749714)=0.02046903$ Rreg, $50 \%=(3.651789-3.727779) /(0-3.749714)=0.02026555$ $\operatorname{Rreg} .60 \%=(3.598993-3.675746) /(0-3.749905)=0.02046799$ Rreg, $70 \%=(3.55108-3.630274) /(0-3.749332)=0.02112216$ $R_{\text {reg, }} 80 \%=(3.486534-3.573816) /(0-3.749905)=0.02 .327579$ $\operatorname{Rreg}_{1} 90 \%=(3.387198-3.496452) /(0-3.750095)=0.02913366$

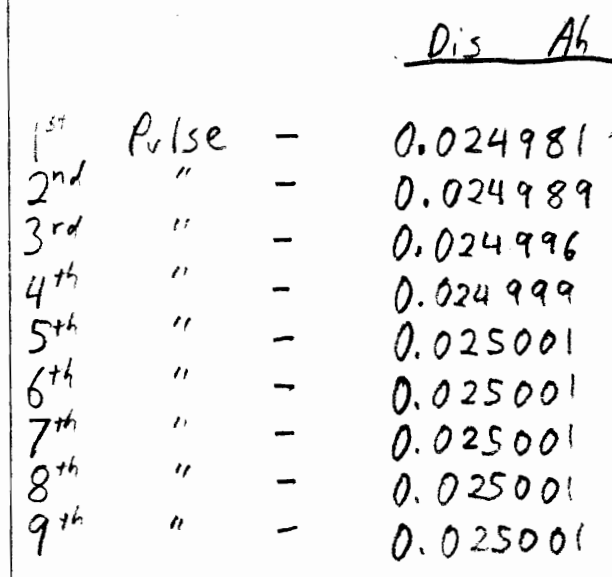

Regen Energy / Ah Pulse Mi) 0.12498 :

z: $\quad 0.224989$

3: $\quad 0.324996$

4: 0.424999

5: 0.525001

6: $\quad 0.625001$

7: 0.725001

8: $\quad 0.825001$

9: $\quad 0.925001$
$10 \%$
0.1
0.1
0.1
0.1
0.1
0.1
0.1
0.1
0.1
0.1

AhI 0.122233

0.222237 0.322241

0.422244

0.522248

0.622252

0.722256

0.82226

0.922263

0.1

01

0.1

0.1

.1

1 listraine

(and

0.1
0.2
0.3
0.4
0.5
0.6
0.7
.0 .8
0.9

Step Ah

0.1

0.2

0.3

0.5

0.6

0.8

0.9 


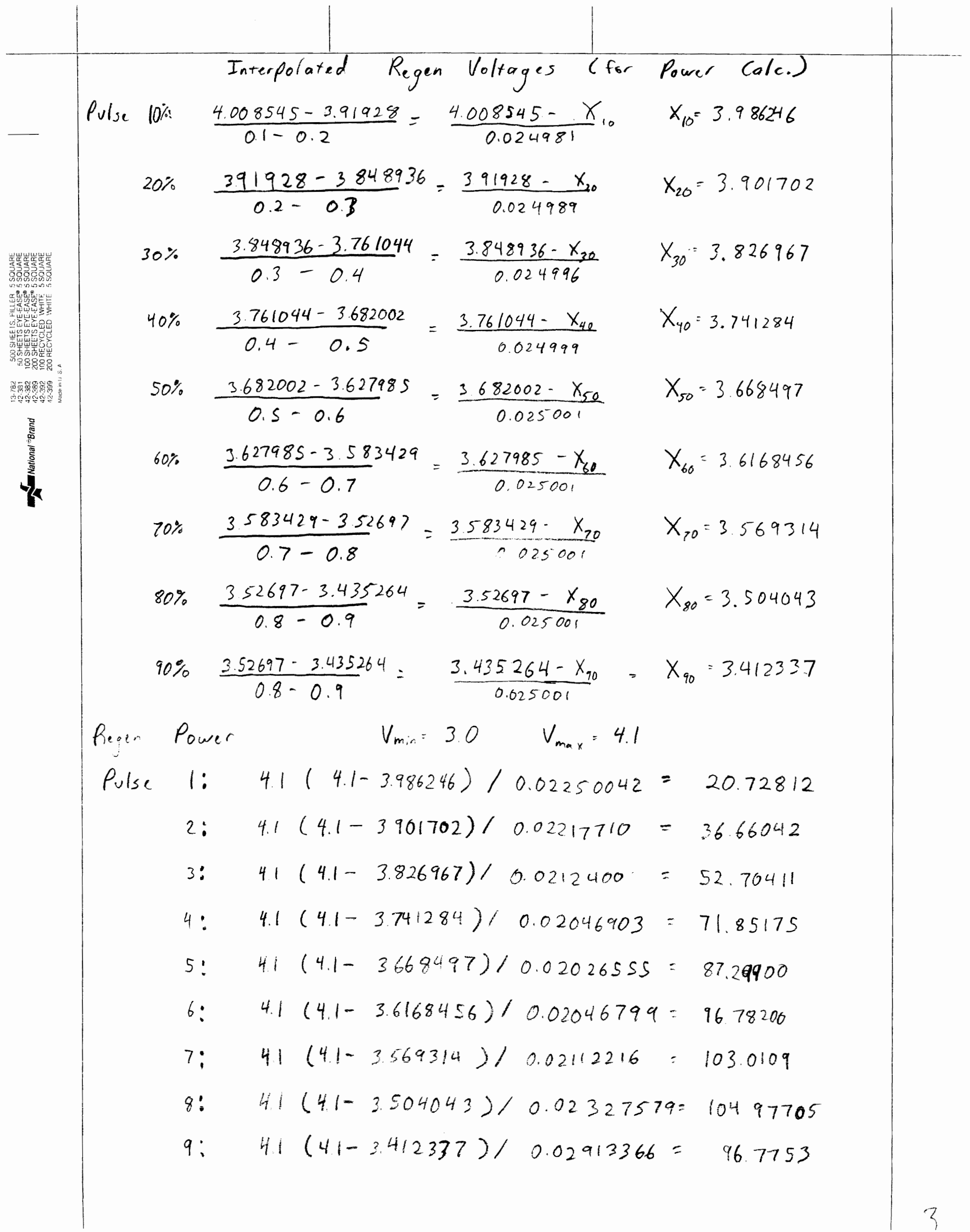




\begin{tabular}{|c|c|c|c|c|c|c|c|}
\hline & \multicolumn{2}{|c|}{ Discharge } & \multicolumn{2}{|c|}{ Power $\quad V_{\min }=3.0$} & \multirow[b]{2}{*}{0.0382086} & \multirow{3}{*}{$\begin{array}{l}= \\
=\end{array}$} & \multirow{3}{*}{$\begin{array}{l}79.18728 \\
74.68680\end{array}$} \\
\hline & Pulse & 1: & $30(4008545$ & $-302 /$ & & & \\
\hline & & $2:$ & $3.0(3.91928$ & -3.001 & 0.0369254 & & \\
\hline & & 3: & $30(3848936$ & $-303 /$ & 0.0370810 & $=$ & 68.68229 \\
\hline taf, & & 4: & $3.0(3.761044$ & $-3.0) /$ & 0.0342399 & $=$ & 66.680452 \\
\hline & & 5 & $3.0(3.682002$ & $-3.0) /$ & 0.0323796 & $=$ & 63.18812 \\
\hline & & 6: & $3.0(3627985$ & $-30) /$ & 0.0328093 & $=$ & 57.42137 \\
\hline 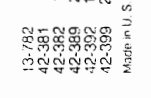 & & $7:$ & $3.0(3.583429$ & $-3.0)$ & 0.0343953 & $=$ & 50.88739 \\
\hline 番 & & 8: & $3.0(3.52697$ & $-30) /$ & 0.0389729 & " & 40.56434 \\
\hline 章 & & $9:$ & $3.0(3.435264$ & $-30) /$ & 0.0734932 & $=$ & 17.76752 \\
\hline
\end{tabular}

Discharge Energy / Ah

\begin{tabular}{|c|c|c|c|c|c|}
\hline \multirow{9}{*}{ Pulse } & & At & Ah 2 & wh1 & wh 2 \\
\hline & D. & $0.1000 \%$ & 0.097232 & 0.400952 & 0.389953 \\
\hline & 0.3 & $\begin{array}{l}0.197236 \\
0.29724\end{array}$ & $\begin{array}{l}0.200014 \\
0.300018\end{array}$ & $\begin{array}{l}0.781308 \\
1.164233\end{array}$ & $\begin{array}{l}0.792054 \\
1.174753\end{array}$ \\
\hline & 0.4 & 0.397243 & 0.400021 & 1.539016 & 1.549313 \\
\hline & $5: 0.5$ & 0.497247 & 0.500025 & 1.906148 & 1.916247 \\
\hline & $6: 0.6$ & 0.597251 & 0.600029 & 2.266818 & 2.276757 \\
\hline & 0.7 & 0.697255 & 0.700033 & 2.622149 & 2.631947 \\
\hline & 0.8 & 0.797259 & 0.800037 & 2.972126 & 2.981757 \\
\hline & 0.9 & $0.81725^{\circ}$ & 0.90004 & 3.314365 & 3.323708 \\
\hline
\end{tabular}

Discharge energy,

Pulse $1 \quad E_{1}=0.400917$

$$
\frac{W_{h 2}-W h 1}{A^{h} 2-A h 1}=\frac{x-W h 1}{A h_{x}-A h l}
$$

$2 \quad E_{2}=0.792008$

$3 \quad E_{3}=1.174702$

$4 \quad E_{4}=1.549252$

$5 \quad E_{5} * 1.916177$

$6 \quad E_{6}: 2.276679$

$7 \quad E_{7}=2.63186$

$8 \quad E_{8}=2.981661$

$E_{q}=3.323609$ 
Regin Energy

Pulse 1

$$
\begin{array}{ll}
2 & E=0.888383 \\
3 & E=1.269069 \\
4 & E=1.641628 \\
5 & E=2.006829 \\
6 & E=2.365926 \\
7 & E=2.71983 \\
8 & E=3.068041 \\
9 & E=3.407134
\end{array}
$$

Regin Power curve $P=8.12 E^{2}+36 E+5.0476$

Discharge Power Curve $\begin{aligned} P= & -8.0899 E^{4}+64.774 E^{3}-19494 E^{2} \\ & +247.7 E-42.992\end{aligned}$

Available Energy (from Graph)

$$
\begin{gathered}
\text { Energy } \\
0 \\
0.479166 \\
1.250 \\
1.833 \\
2.25 \\
2.708
\end{gathered}
$$

\begin{tabular}{c} 
Power- \\
\hline 644 \\
60 \\
50 \\
40 \\
30 \\
20
\end{tabular}

5 


\section{APPENDIX A}

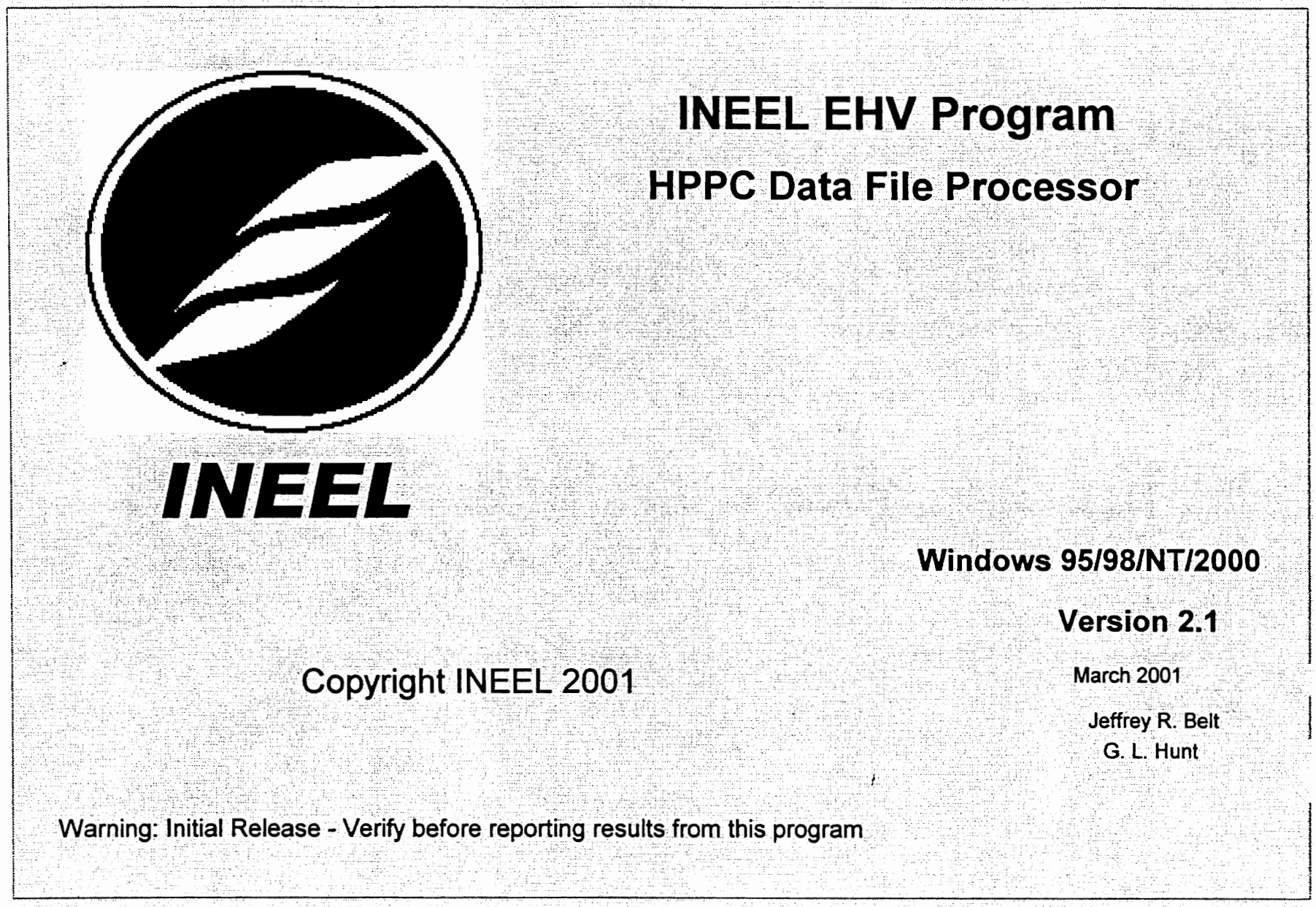




\section{APPENDIX B}

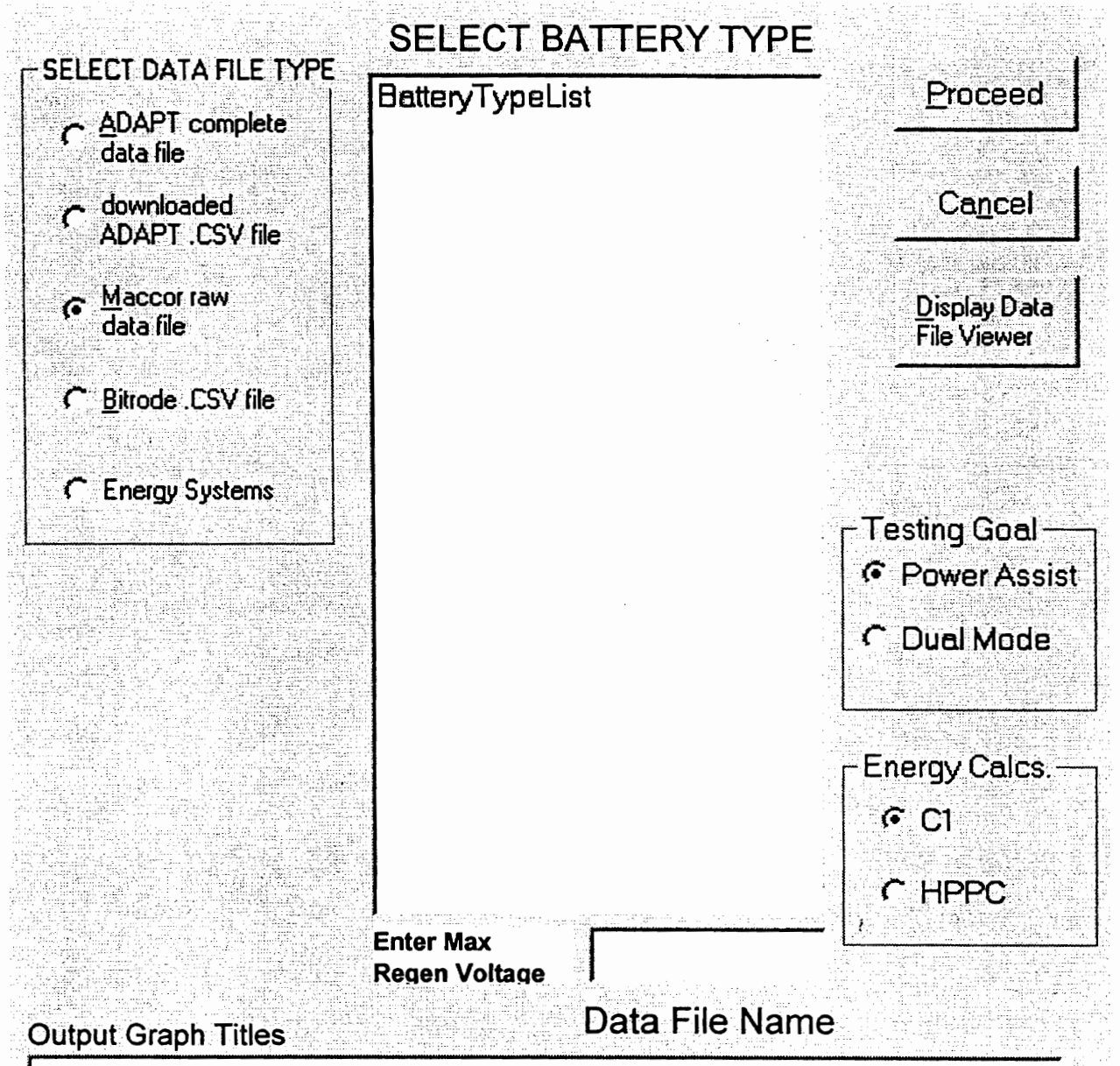




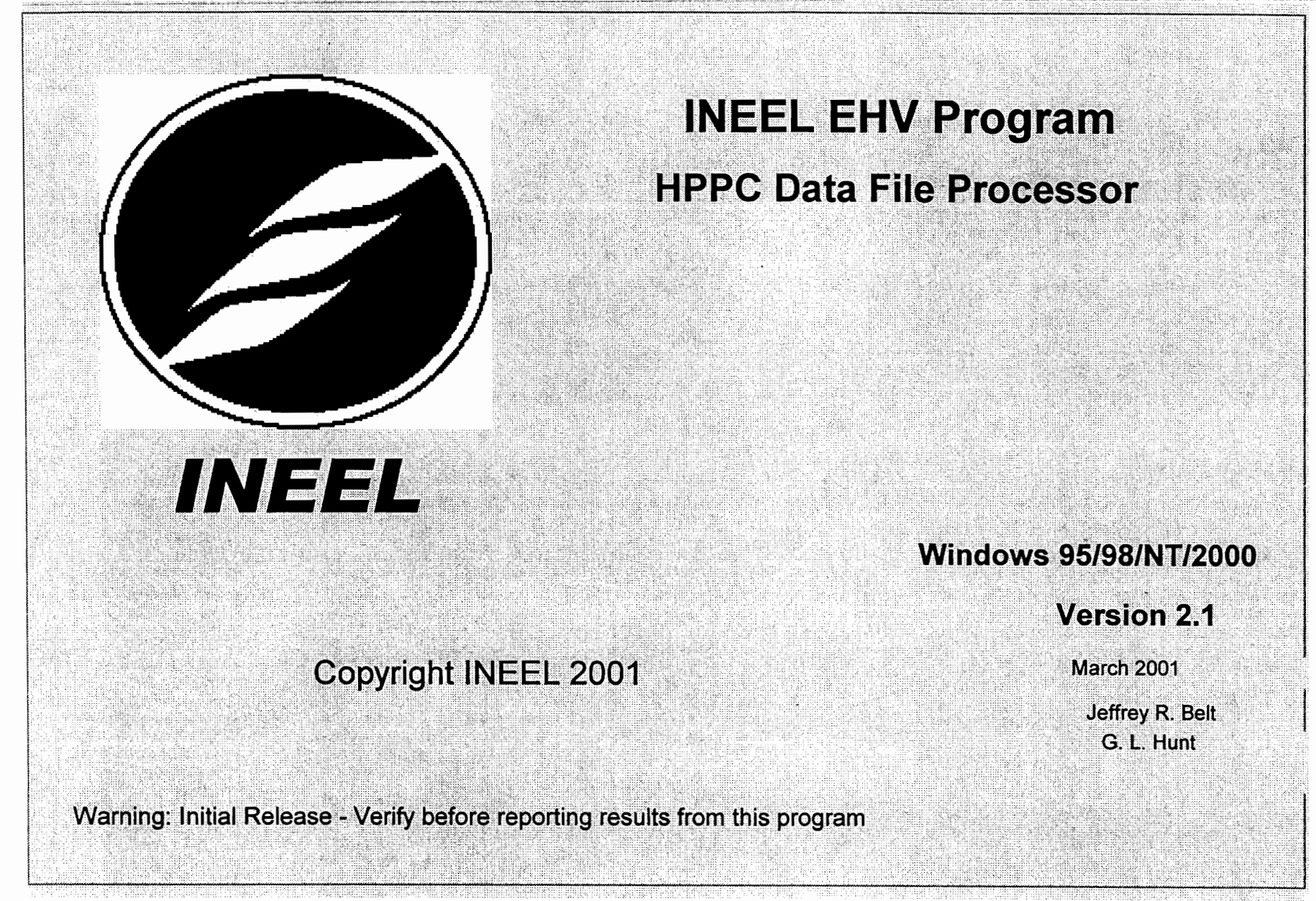




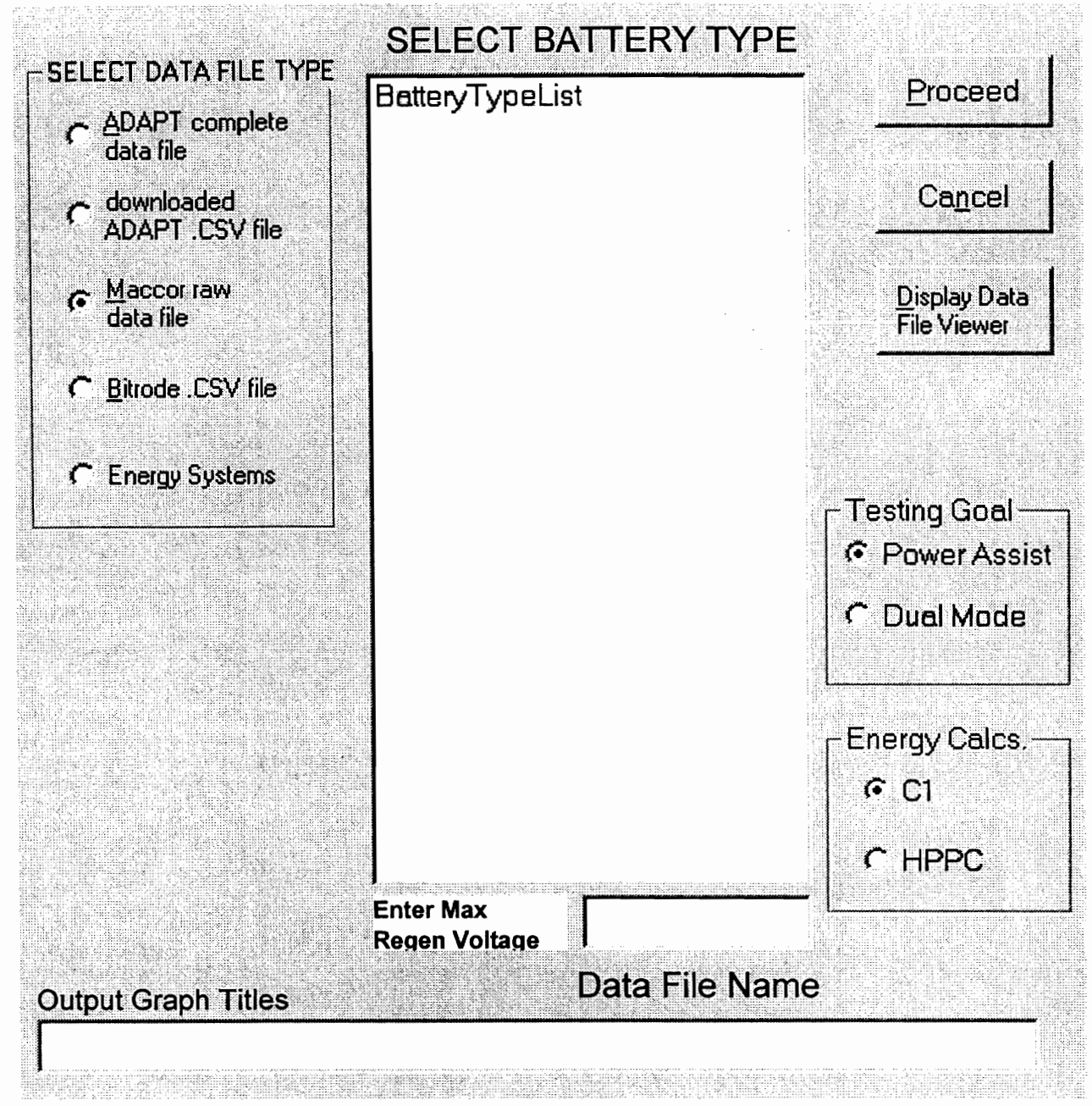


Choose handling of

WRITE to Iext calculation results

fle

Process

ANOTHER

FILE

WRITE to

VIEW Excel

spreadsheet

Spreadsheet

QUIT 